\title{
"Aistifsar" Enquiry Chatbot using Artificial Intelligence Markup Language (AIML)
}

\author{
Shama Rashid Said Al Ghaithi \\ Syed Thoufeeq Ahmed
}

\begin{abstract}
There is always an increase in the number of students enrolling to universities and higher education institutions. During this process the prospective student tend to enquire various particular from university or higher educational institute via telephone, website, emails, friends, postal service and sometimes with agents. The information obtained by prospective student by these means sometimes incomplete and inadequate. Also these means sometimes takes longer time in answering to the queries of prospective student. Moreover institutes are spending huge amount of cost for this purpose as it involve hiring employees, technology and providing the necessary infrastructure. Many commercial establishments with similar problem are trying to address such situation by introducing a "Chatbot" in their web portals or in information systems. A Chatbot is a conversational agent where a computer program is intended to simulate an intelligent discussion or conversation. It can take user contribution to numerous formats like content, voice, and pictures. For this reason, ongoing advances in Artificial Intelligence (AI) have enormously improved the exactness and adequacy of common language preparing and making Chatbot's a practical alternative for query based works. This paper will study the benefits to create a Chatbot for the university and higher educational Institutes and how this technology can be helpful for prospective students to replying to their queries. Also a Chatbot will be developed along with our own dataset for Middle East College, Oman using the principles of Artificial Intelligence Markup Language to know the relevance of such facilities to address the needs of prospective student.
\end{abstract}

\section{Introduction}

The essential conceptualization of the Chatbot is credited to Alan Turing who asked "Can the machines think?" way back in 1950. In the recent years it is observed that human interactions and communication with digital devices is ever increasing and this lead to the development of "Chatbot" (Abdul-Kader, 2015). Researcher also termed chatbots as IM bot, Bot, ACF (Artificial Conversational Entity), chatterbox to name a few. Chatbot is an online exchange process, using computer program, between the human and computers to perform automated tasks. Chatbot uses Artificial Intelligence (AI) techniques to exchange conversation with text and sometimes even with voice. Chatbot have been widely available and used by commercial establishments. Amazon's Alexa, Apple's Siri and Microsoft's Cortana are examples of some of the prominent Chatbots or the virtual assistants. In this paper the usefulness of chatbot for Higher Educational Institute is explored. We have done the project for Middle East College (MEC) in Sultanate of Oman and build a Chatbot, named as "Aistifsar". The core motivation behind Aistifsar is to help addressing the queries of prospective and enrolled students (users) at any time and from anywhere.

\section{For the development of MEC Chatbot the following parameters were considered:}

- For MEC administrative functions how can AI techniques and chatbots can provide support?

- For teaching and learning what role can AI and chatbot play? 
- What cultural challenges can AI and chatbot raise at MEC?

- Can AI and chatbot bring in financial benefits by saving money?

- What are the future prospects of AI and chatbot for MEC?

The MEC Chatbot is developed using AI algorithms which analyses and understand the user's queries. The MEC chatbot is a web application which acts as a plugin for the existing MEC website and MEC portal and hence accessible for user at any given time from anywhere. This saves users and MEC administrative staff's considerable amount of time. The users just need to text in or use the voice recorder facility available in the chatbot to ask the query on MEC activities. The chatbot automatically respond, via same interface, to the user query using the most sophisticated build in AI algorithm. The remaining of the paper is presented in four sections. Following section, section 2 , focus on the some of the state of the art current technologies for chatbots via in depth literature review. Section 3 presents the framework of MEC chatbot. Section 4 presents the developed dataset architecture for MEC chatbot and finally section 5 we present conclusions and way forward.

\section{Background and state of art}

Chatbot is a programming that reacts to regular language info and endeavors to hold a discussion in a way that emulates a genuine individual. Some chatbots are utilized for amusement purposes, and others used it for business and study purposes. Chatbot is getting a considerable measure of consideration from business network right now as they can spare expenses in user benefit focuses and can deal with different users at once. Successful execution of a chatbot calls for right examination of user's inquiry by the bot and the arrangement of the right reaction that ought to be given to the user. In numerous situations the data accessible from the user's inquiry is lacking to give the appropriate response. In such settings, the chatbot should be curious with the goal that it will be more intelligent and can mirror a more common human connection. (Reshmi, 2016) in his work, figure 1, explored report about the inquisitive chatbot, which finds the missing information in inquiry and tests the inquiries to users to gather information that are required to answer the question. Through this execution, the dimension of l evels between the user and the chatbot. 


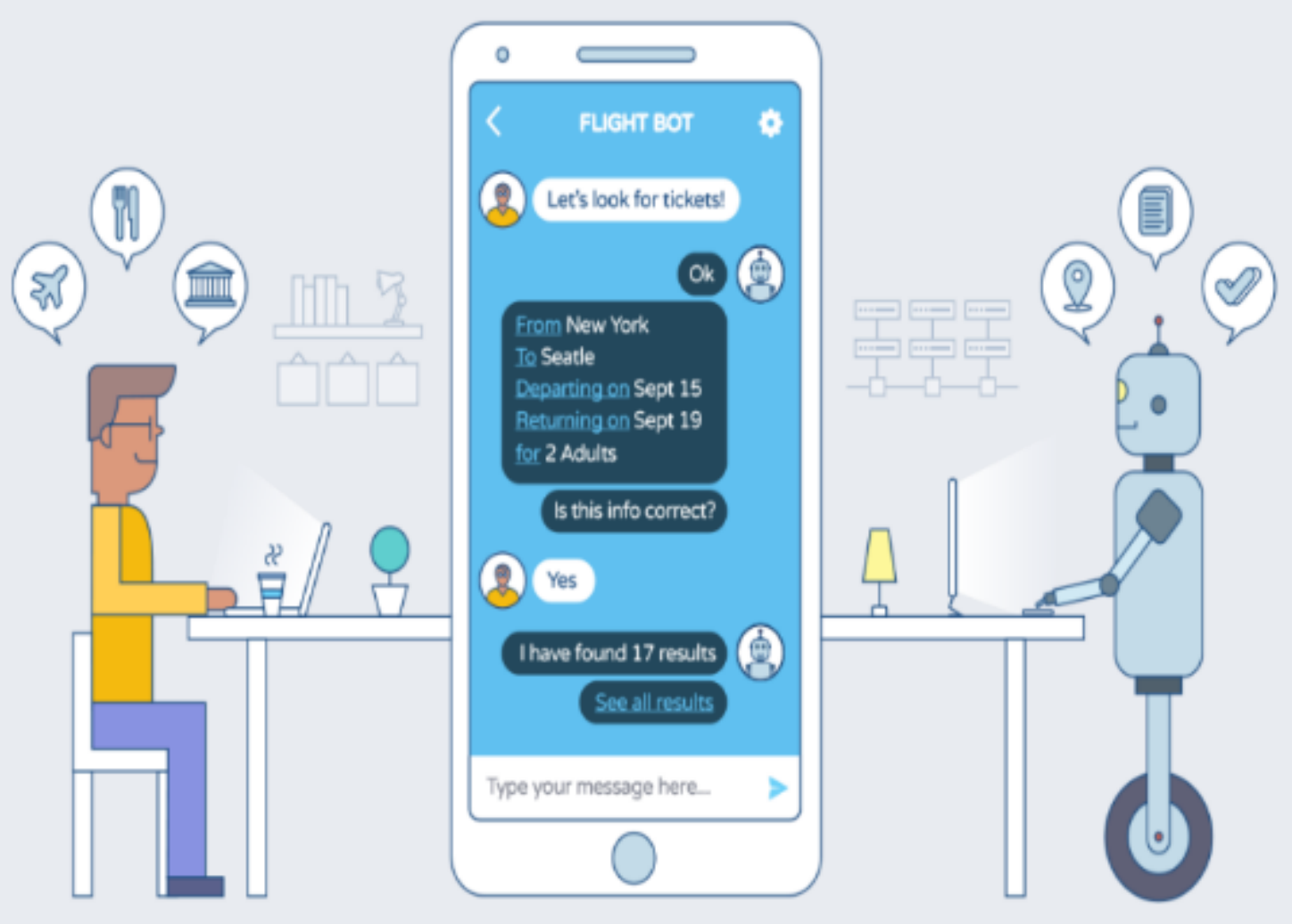

Figure 1. Chatbot Artchitecture of S Reshmi and Balakrishnan

The work of (Efraim, 2017) highlights how to use information from client chatbot discussions, where clients have appraised the responses if it a good or not, they propose an increasingly effective option to a chatbot's keyword based answer recovery. They will test two neural system ways to deal with the close copy question recognition assignment as an initial move towards a superior answer recovery strategy. Convolutional neural system engineering gives promising outcomes on this hard undertaking. (Nevon Projects, 2018) in his research shown the importance of chatbot in higher educational institutes. His work include the investigations of student's inquiries and comprehend student's message. His system is a web application which gives reply to the question of the students. The students simply need to inquiry through the bot which is utilized for chating. Students can talk utilizing any format there is no explicit arrangement the user needs to use. The System utilizes artificial intelligence to answer the questions. The responses are suitable to the student questions. The student can ask about anything the college through the system. And does not need to by go to the college for enquiry. The student can question anything's college related for example, date and timing of yearly day, specialization in the college, and study offers. This system causes the student to be learned about the about the college information.

Advanced education specialists (Putz, 2017) are anticipating chatbots to be the future of correspondence among colleges and students. For some colleges, enhancing correspondence with students has been a key interest. As any student knows, the way researching about the college specializations can be a complex. Regardless of whether it's talking with different people and divisions, perusing through program pages or discovering the correct data for money related guide, the measure of research required to choose the correct college can be daunting. So, with developing chatbot innovation, colleges conveying data to students. Also chatbot will be one of the possible way for marketing and spreading information about the colleges. (Pichponreay, 2016) has presented different learning styles of users, their process of chatbot is shown figure 2 . With fast 


\section{Journal of Student Research}

advancement of data and correspondence innovation, individuals are extremely different in learning style, and information enhancement strategies. This article shows a methodology of changing over records into information of Chatbot system that empowers clients to make more advantages of it by asking and noting inquiries using electronic reports incorporated with recreate system. It is a coordinated system for enhance substance of archives from famous configuration, for example, Portable Document Format (PDF) and advanced photographs. The work process of this system is begun from concentrates writings utilizing Optical Character Recognition (OCR) from records, at that point produces questions by means of over generating Transformations and Ranking calculation, lastly let Chatbot reaction to the client's inquiry when it is coordinated with the String pattern.

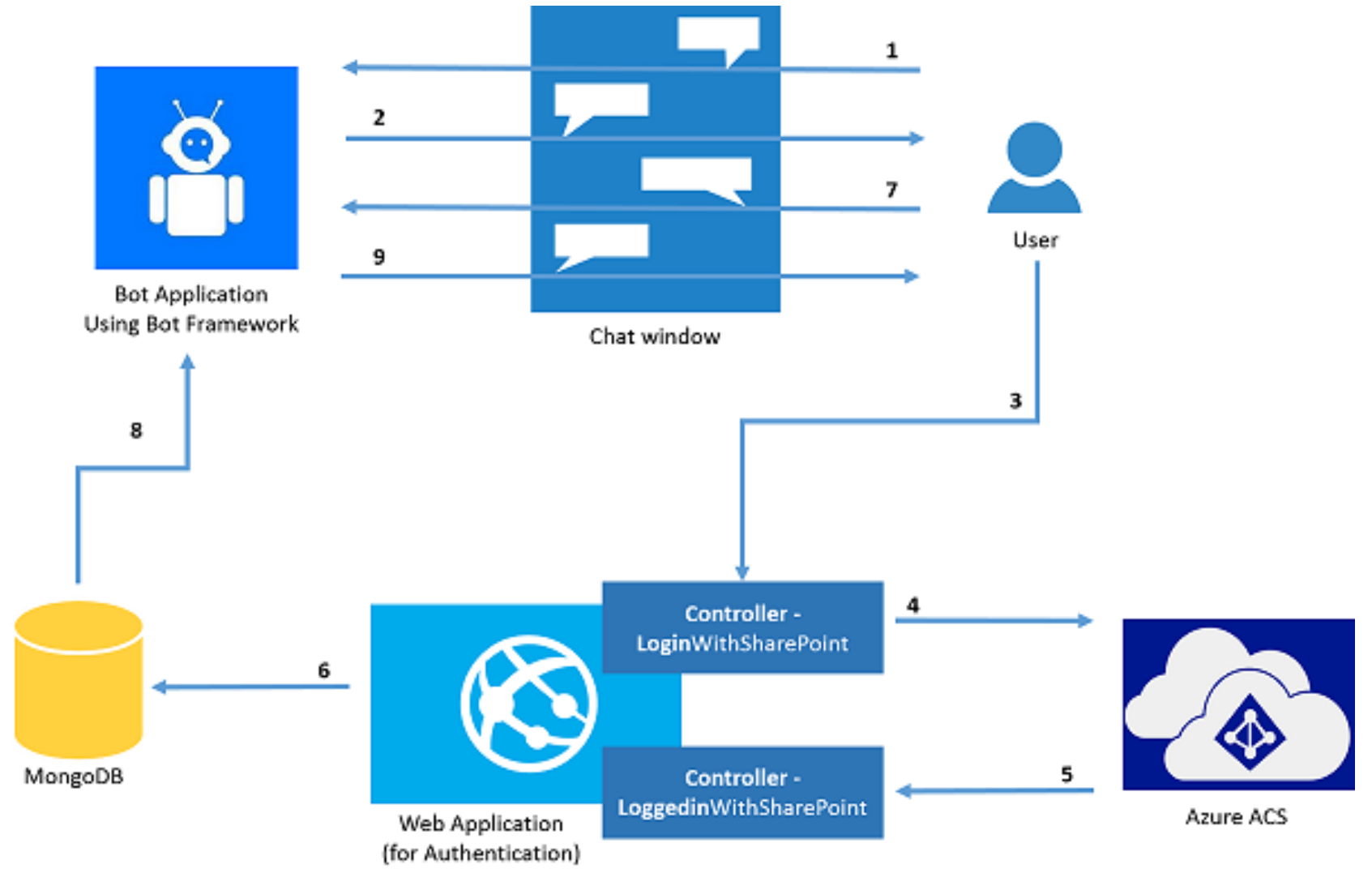

Figure 2. Advanced process of chatbot for different learning styles of user

\section{Framework of Aistifsar Chatbot}

The methodology used for this project is iterative software development model. Workflow of Wechat (WeChat AI NLP, 2017), figure 3, is used to develop the MEC chatbot. 


\section{Journal of Student Research}

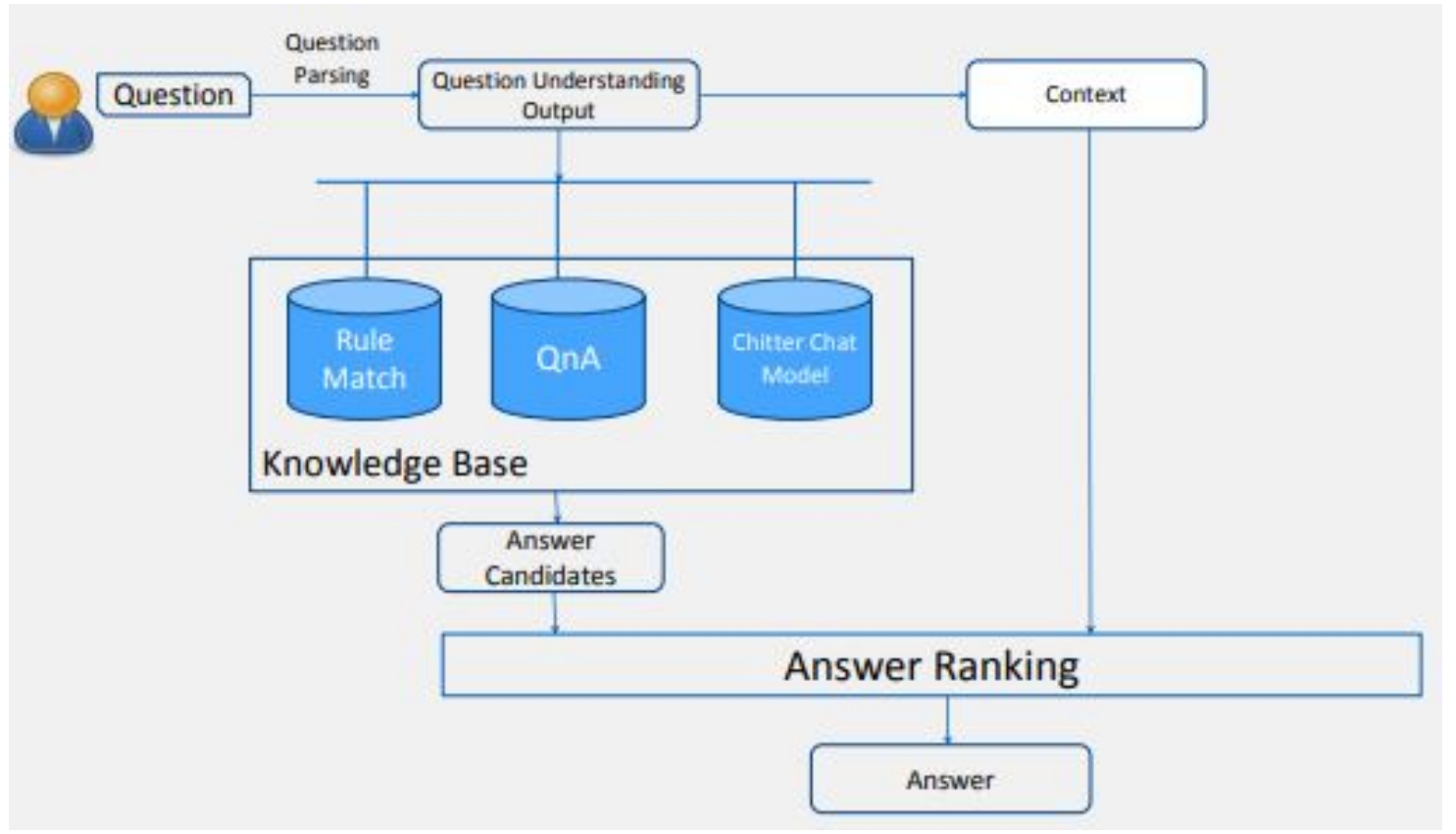

Figure 3. Workflow of Wechat chatbot

The context diagram of MEC chatbot is given in figure 4.

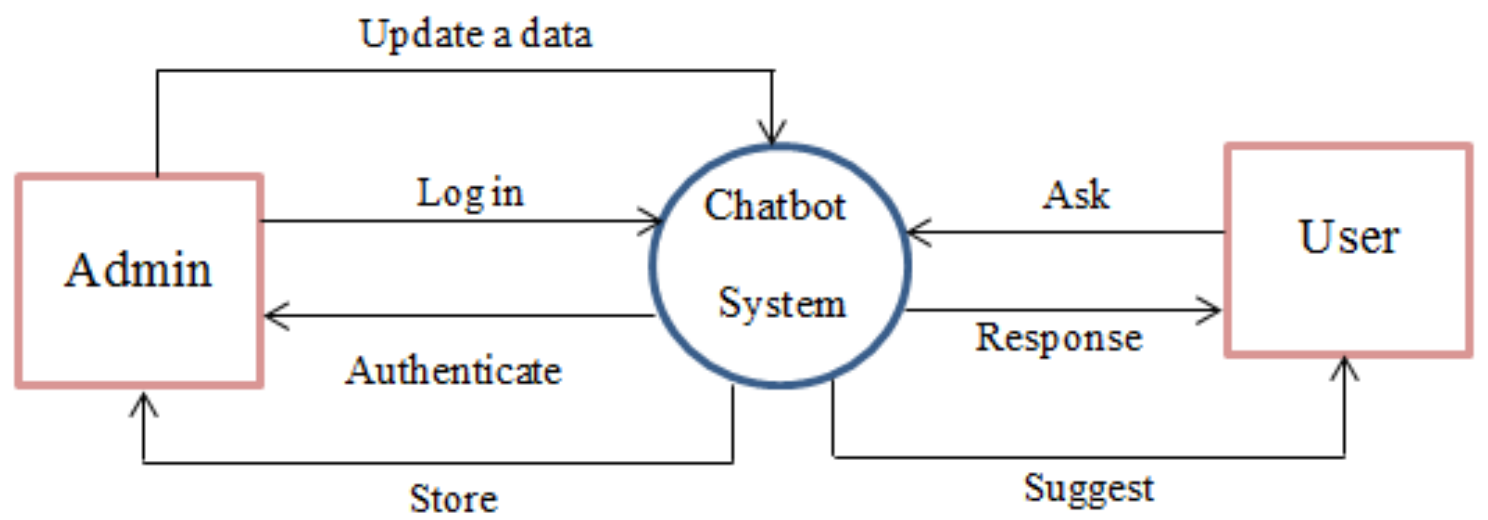

Figure 4. Context diagram of $M E C$ chatbot

The context diagram depicts the procedure of each outer/external element of the system. The outside elements that utilize the system are admin and user. Context diagram also demonstrates a portion of the procedures for each entity with respect to the system and what the system is giving and providing for everyone in the meantime. The system enable the admin to get to the system to include, delete, update, and scan for the questions and answers data. Also, produce a report. The admin can sign in to the system and add new question with the answer and answer for the user question that not has the answer. Also, enter the suggestion. The user can open the system and start to ask in the chat. Figure 5 shows the class diagram of MEC chatbot. The MEC chatbot class diagram static perspective on an application. It is not utilized for imagining, depicting, and documenting various parts of a system but in addition to building an executable code of the product application 


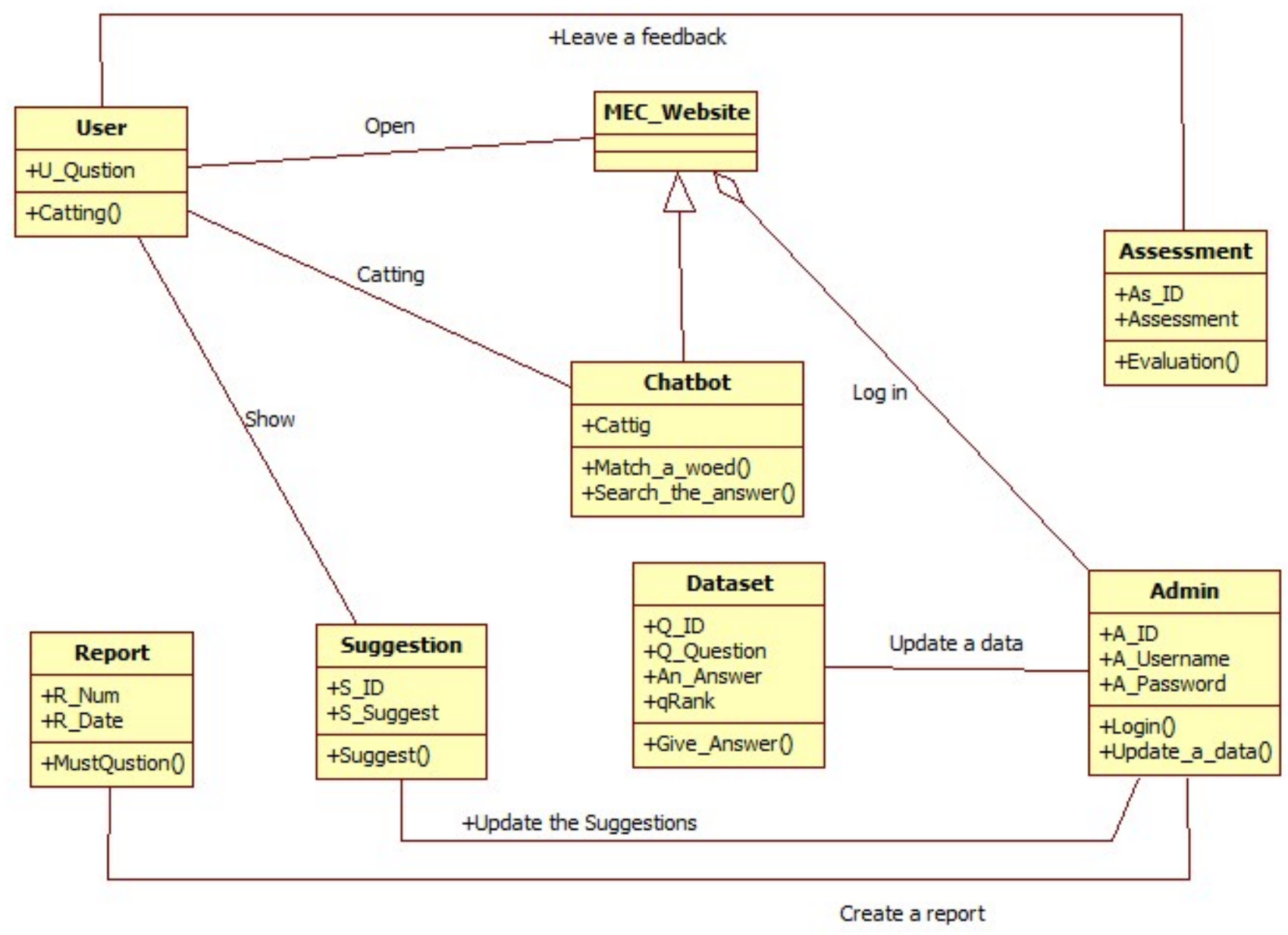

Figure 5. MEC chatbot class diagram

The MEC chatbot has eight classes: admin, user, dataset, report, Chatbot, MEC website, assessment, and suggestion. Each class have functions and attributes. Also relationship between each class: association, aggregation, generalization, and realization. MEC chatbot holds the following relationships:

- Between user and MEC_website the association relationship. (Open)

- Between user and Chatbot the association relationship. (Chatting)

- Between user and assessment the association relationship. (Leave a feedback)

- Between admin and dataset the association relationship. (Update a data)

- Between admin and suggestion the association relationship. (Update a suggest)

- Between admin and report the association relationship. (Create a report)

- Between admin and assessment the association relationship. (Show the assessment)

- Between admin and MEC_website the aggregation relationship. (Log in)

- Between Chatbot and dataset the association relationship. (Match) 


\section{Journal of Student Research}

Fourth Middle East College Student Research Conference, Muscat, Sultanate of Oman

- Between Chatbot and dataset the realization relationship. (Search the answer)

- Between Chatbot and MEC_website the generalization relationship.

- Between Suggestion and Chatbot the association relationship. (Send suggest).

A few screenshots of the developed MEC chatbot are given in figure 6 and figure 7 .

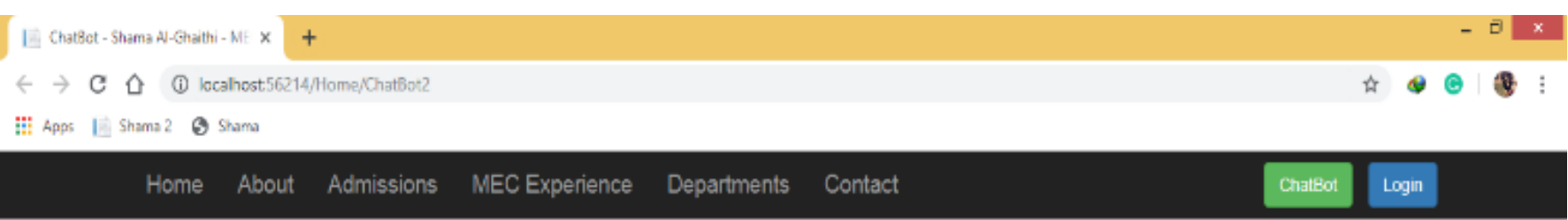

\section{Welcome to MEC ChatBot}

\section{Open ChatBot}

\section{(C) 2019 - Mrs. Shama Rashid Al-Ghaithi (ID NO.:14F12339), Under Supervision of Dr. Thoufeeg Ahmed Syed}

Figure 6. Home page of MEC chatbot 


\section{Journal of Student Research}

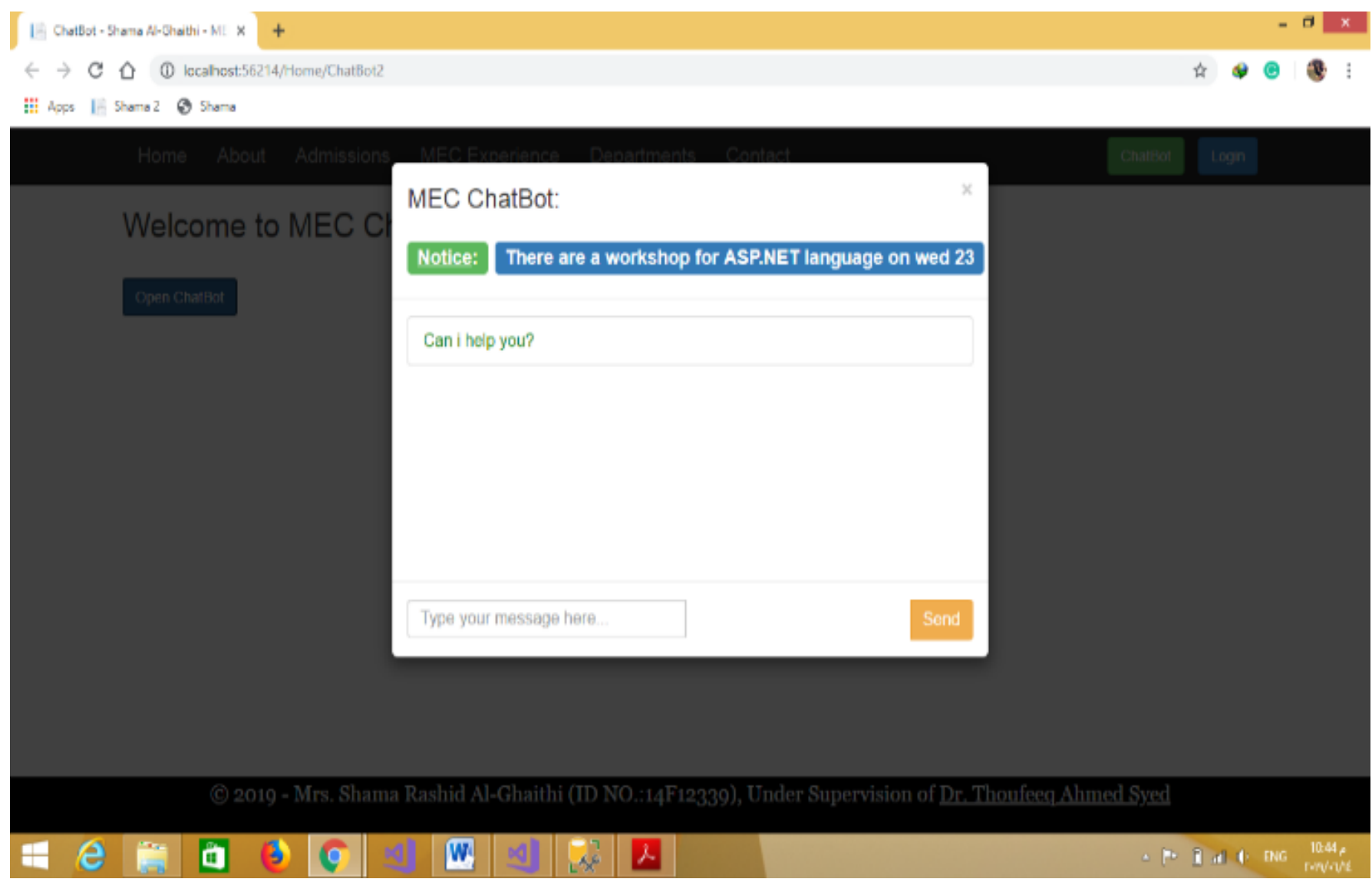

Figure 7. MEC chatbot interaction page

\section{Dataset Architecture}

In this section, and as seen in figure 8 we build the dataset for the MEC Chatbot to store all the data in separate database. The dataset is simply gatherings of information and can be tied in with anything. For example, you could have datasets of the college, college system, inquiries, and answer. Datasets are a necessary piece of AI and natural language processing on the grounds that you can utilize them to prepare models to do assignments, for example, extract texts, text arrangement, and item classification. Dataset was built by considering four parameters: 1 . List of the all the possible queries. 2. User: This database table for the admin allowing to update data for the chatbot when needs. 3. List of suggestions, for the users listing all the scheduled events and workshops at MEC. 4. List of feedback to get the user's opinion to improve the system.

When the user visits the MEC website, user can start the chatbot and start the conversational with a computer. The user will write a query or text in the textbox area of chatbot and then chatbot will analyze the words of the query to match it with the list answer from the dataset to provide the most matching answer to the user query and if the chatbot didn't find the matching answer or no match for the query chatbot will save the question in the list of question tables and alerted the admin who can to see the new query and update the answer for future references. Also when the user finishing the conversation can give feedback about the chatbot where the result of the feedback will be stored in a database table. The parameters used where user can give the feedback are excellent, very good and good. Also the chatbot dataset can store and list the frequently repeating queries. A provision to list top ten queries has provided in the MEC chatbot. 


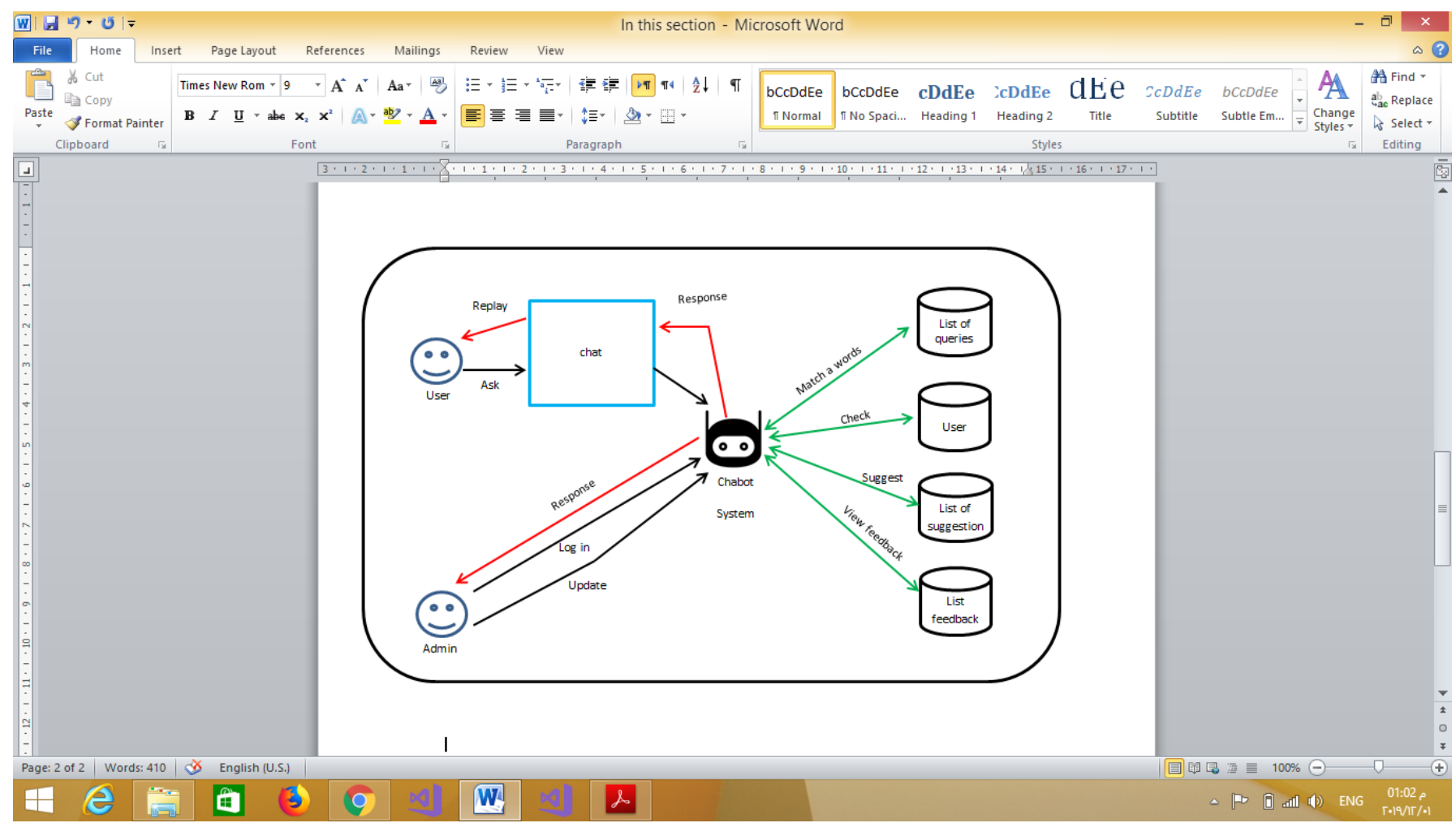

Figure 8. Chatbot Dataset Architecture

\section{Conclusions}

In this paper, an automated chatbot is presented for MEC. The systems uses state of the art chatbot frameworks and AI algorithms. A developed chatbot is used as a plugin on MEC website and MEC portal. Though the system solve the basic queries of users using the developed dataset, dynamic updating the dataset in real time scenario is a challenge.

The way forward is to look for the opportunities that can be included in MEC chatbot. This may include, but not limited to, 1. Can the chatbot be used as peer tutor? 2. Can the chatbot can be used in formative assessment for providing feedback? 3. Can chatbot be used to reduce the overall administrative work of academic and nonacademic staff?

\section{References}

Abdul-Kader, S.A. and Woods, J.C., 2015. Survey on chatbot design techniques in speech conversation systems. International Journal of Advanced Computer Science and Applications, 6(7).

Reshmi, S. and Balakrishnan, K., 2016. Implementation of an inquisitive chatbot for database supported knowledge bases. sādhanā, 41(10), pp.1173-1178.

Efraim, O., Maraev, V. and Rodrigues, J., 2017, September. Boosting a rule-based chatbot using statistics and user satisfaction ratings. In Conference on Artificial Intelligence and Natural Language (pp. 27-41). Springer, Cham.

Nevon Project "College Enquiry Chat Bot"[Online] aviolable on http://nevonprojects.com/collegeenquiry-chat-bot/ [accessed on 20 Nov 2018].

Putz, T., 2017. Higher Education Marketing: Why Chatbots are the Future of Communication. 


\section{Journal of Student Research}

Fourth Middle East College Student Research Conference, Muscat, Sultanate of Oman

Higher Ed Marketing Journal, 6th September 2017.

Pichponreay, L., Kim, J.H., Choi, C.H., Lee, K.H. and Cho, W.S., 2016, July. Smart answering Chatbot based on OCR and Overgenerating Transformations and Ranking. In 2016 Eighth International Conference on Ubiquitous and Future Networks (ICUFN) (pp. 1002-1005). IEEE.

WeChat AI NLP, 2017 "Intelligent Chatbot on WeChat" available on http://ondemand.gputechconf.com/gtc/2017/presentation/s7630-niu-intelligent-chatbot-wechat.pdf. [accessed on 25 Nov 2018]. 